\title{
Le bois d'aloès, « le Bois des Dieux »
}

La vie d'un arbre blessé

Agarwood, "The wood of the Gods". The life of a wounded tree

\section{Gerard A. Persoon}

\section{(2) OpenEdition}

Journals

Édition électronique

URL : http://journals.openedition.org/ethnoecologie/704

DOI : $10.4000 /$ ethnoecologie.704

ISSN : 2267-2419

Éditeur

Laboratoire Eco-anthropologie et Ethnobiologie

Référence électronique

Gerard A. Persoon, «Le bois d'aloès, «le Bois des Dieux » », Revue d'ethnoécologie [En ligne], 1 | 2012, mis en ligne le 30 juin 2012, consulté le 19 avril 2019. URL : http://journals.openedition.org/ ethnoecologie/704; DOI : 10.4000/ethnoecologie.704

Ce document a été généré automatiquement le 19 avril 2019

\section{(c) $(1) \& \Theta$}

Revue d'ethnoécologie est mis à disposition selon les termes de la licence Creative Commons Attribution - Pas d'Utilisation Commerciale - Pas de Modification 4.0 International. 


\section{Le bois d'aloès, « le Bois des Dieux »}

La vie d'un arbre blessé

Agarwood, "The wood of the Gods". The life of a wounded tree

Gerard A. Persoon

\section{Introduction}

1 L'idée de prendre pour thème de réflexion le bois d'aloès, que l'on appelle aussi souvent «Le Bois des Dieux», a germé lorsqu'un négociant en bois d'aloès à Samarinda (Kalimantan oriental) m'a expliqué quelles étaient les différentes destinations des stocks dans sa petite réserve. Certains sacs de bois d'aloès devaient partir pour Bali pour y être utilisés dans des rituels hindouistes, les grosses pièces massives seraient livrées à Singapour d'où elles seraient réexportées vers le Japon pour servir à la fabrication d'encens de haute qualité, des prêtres catholiques locaux collecteraient de petits sachets alors que les négociants arabes seraient intéressés par un type spécifique de bois d'aloès pour la distillation de l'huile utilisée pour l'encens destiné aux rituels musulmans ainsi que pour la fabrication de parfums. Une partie du bois d'aloès exporté à Singapour serait redirigée sur la Chine, Taiwan, la Corée et la Thaillande et utilisée par les bouddhistes dans des cérémonies, sous une forme ou sous une autre.

2 Mais cette fonction religieuse spéciale implique-t-elle un traitement particulier de l'arbre en termes de protection? L'arbre dont est issu le bois d'aloès a-t-il un statut de «plante sacrée ", ou peut-on le considérer comme un exemple de protection de l'environnement inspiré par le religieux?

\section{Le bois d'aloès}

3 Moines bouddhistes, parfumeurs arabes, fabricants japonais d'encens et récoltants de bois d'aloès de Papouasie-Nouvelle-Guinée et du Laos ne représentaient qu'une faible partie des personnes qui assistaient au deuxième congrès international du bois d'aloès (mars 
2007, Bangkok). Les participants venaient de plus de trente pays. Le «monde scientifique » était représenté par des pathologistes du bois, des anthropologues, des forestiers, des économistes et des analystes de laboratoire ayant chacun leurs intérêts de recherche particuliers. Outre les scientifiques, on trouvait des entrepreneurs australiens, des investisseurs potentiels dans les opportunités offertes par les plantations d'Aquilaria. Enfin, il y avait les conservateurs de la nature, attachés à la survie de cette espèce d'arbre. Plus de cent vingt personnes au total (couvrant l'éventail complet de la production à la consommation du bois d'aloès), se sont retrouvées pour discuter de l'existence future du bois infecté d'un arbre blessé.

Photo 1. Le flair est encore le meilleur critère pour déterminer la qualité du bois d'aloès et des produits qui en dérivent

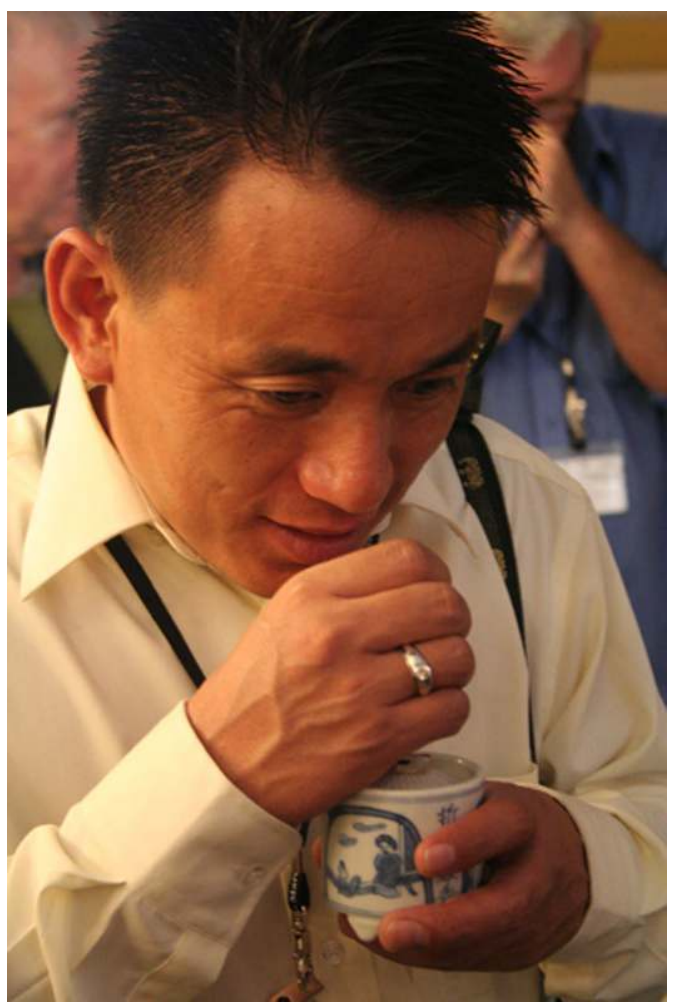

Cliché G.A. Persoon

4 Pendant toute une semaine, ils ont discuté sur les résultats de leurs recherches et de leurs expériences. Des visites de plantations, de distilleries et d'entreprises commercialisant le bois d'aloès ont complété le programme. Au fil de la semaine, des comparaisons fascinantes ont été réalisées entre les résultats d'expériences effectuées en laboratoire et les opinions de négociants qui font encore largement confiance à « l'œil nu et à l'oreille » pour déterminer la qualité du matériau. Cette dernière méthode offre un contraste saisissant avec l'état actuel des découvertes scientifiques sur le terrain de la microbiologie, de l'analyse des bioréacteurs et des empreintes d'ADN. En marge des sessions plénières, avaient aussi lieu des discussions en petit cercle sur les secrets professionnels concernant les meilleures sources de bois d'aloès, sur l'art de l'élaboration des parfums et la fabrication de l'encens, et sur la persistance du commerce illégal. Entre les débats avaient également lieu des séances d'essai de «nez » et des tests de produits de bois d'aloès. 


\section{Histoire du bois d'aloès et ses usages}

5 Le bois d'aloès est le cœur du bois d'un certain nombre d'espèces d'Aquilaria d'Asie du Sud-Est, l'Indonésie, la Malaisie, le Vietnam, le Cambodge, la Thaïlande, le Laos et la Papouasie-Nouvelle-Guinée en étant les principaux pays producteurs et Singapour le centre essentiel du commerce. Le cœur du bois imprégné de résine est parfumé, et donc très recherché et précieux. La résine est produite par l'arbre en réponse à un processus pathologique ou à une blessure. On pense aussi que la production de résine est une réaction à une infection fongique. Ce qui est intéressant, toutefois, c'est que tous les Aquilaria ne produisent pas de résine, et qu'il est extrêmement difficile (et même impossible) de juger de l'extérieur si un arbre est ou non infecté. Il paraîtrait pourtant que certains spécialistes emploient des techniques permettant de différencier les arbres infectés et non infectés sans avoir à les abattre (Donovan \& Puri 2004 ; NTFP Exchange Programme, 2006). Dans la plupart des cas, couper l'arbre est le seul moyen de se rendre compte s'il contient de la résine.

6 L'usage du bois d'aloès est attesté dans nombre de cultures anciennes, bien que l'histoire de cet usage doive encore être écrite. Les anciens Égyptiens auraient utilisé de l'encens de bois d'aloès dans leurs rites funéraires il y a plus de trois mille ans. Il a aussi été suggéré que le commerce de l'encens a constitué en fait la première route de négoce international de l'histoire. Au Japon, le bois d'aloès serait arrivé avec le bouddhisme. De même, au Vietnam, des textes anciens font aussi référence à l'utilisation de bois d'aloès en relation avec des moines bouddhistes itinérants. Des ouvrages sur l'époque coloniale en Asie du Sud-Est (Indonésie et Malaisie) mentionnent souvent le bois d'aloès comme produit forestier non ligneux très important (voir, par exemple, Schuitemaker 1933 et Dunn 1982).

7 Aujourd'hui, l'éventail des produits tirés du bois d'aloès et de leurs usages est vaste. Au Japon, en Corée et à Taiwan, on considère des pièces massives de ce bois à l'état naturel comme « de l'art brut » hautement apprécié. Des artisans transforment les pièces de bois d'aloès brut en belles sculptures. Le bois est aussi tourné en perles et en bracelets. La plus grande partie du bois, cependant, est transformée soit en huile utilisée dans les parfums et autres produits cosmétiques, soit en copeaux qui sont ensuite réduits en poudre, ingrédient de base de la fabrication d'encens (et parfois aussi de cigarettes spéciales). L'huile est aussi un élément entrant dans l'élaboration de la pharmacopée traditionnelle chinoise et coréenne, dans la préparation de vin médicinal et d'autres produits variés.

L'huile est surtout employée dans le monde arabe où elle est très demandée. Il s'agit de la plus précieuse des huiles essentielles, dont le prix atteint jusqu'à dix fois celui de l'huile de bois de santal. Le plus grand marché pour l'encens de qualité supérieure est le Japon, qui connaît une longue tradition dans la fabrication de l'encens. Les pays arabes, de même que le Japon, s'intéressent au bois d'aloès de haute qualité et les fabricants préfèrent transformer la matière brute eux-mêmes, ceci afin d'éviter que le bois de haute qualité ne soit mélangé à du bois de qualité inférieure.

9 L'huile est extraite du bois par distillation. Ce processus délicat détermine aussi bien la quantité que la qualité de l'huile. Le bois est pilé en tout petits morceaux et/ou en poudre, puis immergé dans l'eau et laissé à fermenter un certain temps. Le mélange est ensuite transféré dans des alambics et chauffé à la vapeur. L'eau, qui s'est condensée sous l'effet 
de la chaleur, et l'huile sont transférées dans un récipient dans lequel l'huile flotte à la surface. Après avoir enlevé l'eau du récipient on peut en extraire l'huile. Le prix d'une huile de haute qualité peut atteindre 50000 à 80000 dollars le litre. Le processus peut être répété une ou deux fois selon la qualité de l'eau et le coût de la distillation. La poudre qui reste après la distillation peut être utilisée pour la fabrication d'encens de qualité inférieure ou de produits parfumés. On estime que cent à cent cinquante kilogrammes de bois d'aloès sont nécessaires à la fabrication d'un litre d'huile.

Photo 2. Séparation des copeaux et de la poudre de bois d'aloès au Kalimantan Oriental, avant de poursuivre le processus de fabrication

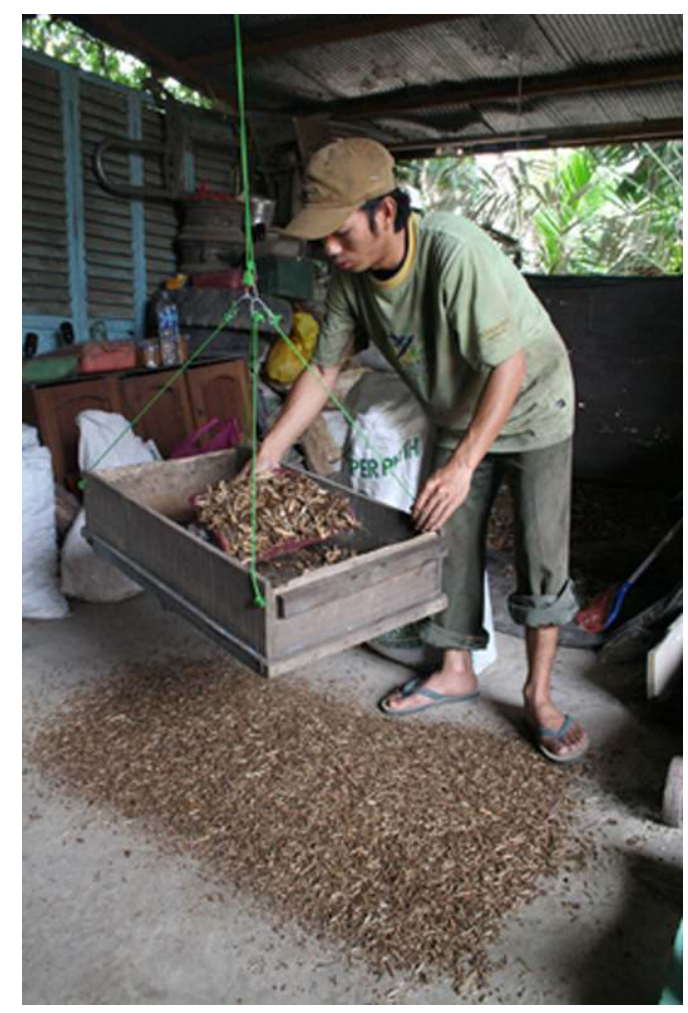

Cliché G.A. Persoon

L'usage le plus important du bois d'aloès est sans aucun doute celui qui en est fait dans un but religieux. L'encens est utilisé dans un large éventail de religions. En fait, l'encens joue un rôle important dans presque toutes les religions par son parfum plaisant, sa fumée qui s'élève vers le ciel, sa nature purifiante du fait de son rapport avec le feu et d'autres raisons variées. Parmi les fonctions symboliques de l'encens qui sont mentionnées dans la littérature au sujet de son usage religieux, on trouve :

- rendre hommage à la divinité, aux dieux ou aux ancêtres et autres puissances spirituelles ;

- stimuler l'ardeur des fidèles ;

- accompagner les prières qui s'élèvent vers le ciel ;

- accompagner les sacrifices faits au ciel ;

- en relation avec les rites funéraires, assurer le passage du défunt vers l'autre monde ;

- purifier/préparer les icônes religieuses, autels et officiants ; (Hoskin 1994; Groom 1981; Catholic Encyclopedia 1998).

Par comparaison avec l'attention portée à l'utilisation et à l'importance de l'encens dans le symbolisme religieux, il est surprenant de constater combien on s'attache peu à la 
question de la production des végétaux qui donnent l'encens, y compris les Aquilaria. Parmi les autres végétaux à la base de la production d'ingrédients pour l'encens on trouve l'oliban (ou gomme de frankincense), le bois de santal, le benjoin, le camphre, le jasmin et le patchouli. Dans les publications, on trouve peu de choses, sinon rien, sur la production elle-même, les personnes impliquées dans la récolte, ou le statut des arbres. Les matériaux tirés du bois d'aloès entrant dans la composition de l'encens proviennent, dans la plupart des cas, de réseaux commerciaux complexes qui comprennent des négociants récoltants qui opèrent à la frontière des aires de récolte, des grossistes et des distributeurs. Qualité et prix sont les facteurs dominants, mais, étant donné la nature du produit, il est évident que la confiance entre vendeurs et acheteurs est d'une importance capitale. Seule une proportion minime d'utilisateurs cultive et récolte le bois d'aloès ellemême. Les moines bouddhistes, au Vietnam et en Thaïlande, ont en général des Aquilaria dans le jardin entourant leur monastère pour subvenir à leurs besoins, qui sont limités. Dans d'autres cas, les besoins sont comblés grâce à des réseaux commerciaux entièrement fondés sur des principes économiques.

Tous les acteurs impliqués dans la chaîne commerciale reconnaissent qu'une grande partie des affaires se font de façon illégale. Il arrive souvent que les conditions requises par la CITES $^{1}$ ne soient pas remplies. Les conditions de durabilité de la production du bois d'aloès ne semblent pas être une préoccupation majeure pour la plupart des marchands et des consommateurs. Il n'existe pas à ce jour de système de certification pour le bois d'aloès comparable, par exemple, avec un label $\mathrm{FSC}^{2}$ pour le bois d'œuvre issu de la production durable.

\section{Bois d'aloès sauvage et cultivé}

Pendant des siècles, le bois d'aloès a été récolté dans la nature. Traditionnellement, on coupait les Aquilaria et on cherchait le bois infecté au creux de l'arbre. Le prix du bois d'aloès de haute qualité peut atteindre 1000 dollars US le kilogramme. Le commerce s'organisait en fonction d'une frontière qui se déplaçait constamment et pénétrait dans la forêt tropicale des basses-terres d'Asie du Sud-Est. Les négociants étaient continuellement à la recherche de forêts intactes où poussaient des Aquilaria. Le prix des arbres était élevé et, en conséquence, les nouvelles concernant la découverte de bois d'aloès se répandaient comme la « fièvre de l'or ». Des sommes d'argent importantes et des biens de luxe de toutes sortes étaient offerts aux communautés habitant la forêt en échange de bois d'aloès. Récemment, ce type de «fièvre du bois d'aloès " s'est répandue dans les forêts du Kalimantan oriental, de la Papouasie-Nouvelle-Guinée et du Laos (Momberg et al. 2000 ; Wollenberg 2001 et 2003 ; Zich \& Compton 2001). D'habitude, cette " fièvre» était temporaire. Les plus grands arbres une fois coupés, les nouvelles prospections donnaient de moins bons résultats, et, comme dans le cas de l'or, la collecte de petites quantités de bois d'aloès rendait cette activité moins rentable. Cependant, cette méthode de récolte a mené à une exploitation de la ressource au-delà des limites de l'écologie reproductive des arbres d'Aquilaria (Soehartono \& Newton 2001). 
Photo 3. Des blessures expérimentales des arbres en Thaillande pour obtenir la production maximale de résine du bois d'aloès

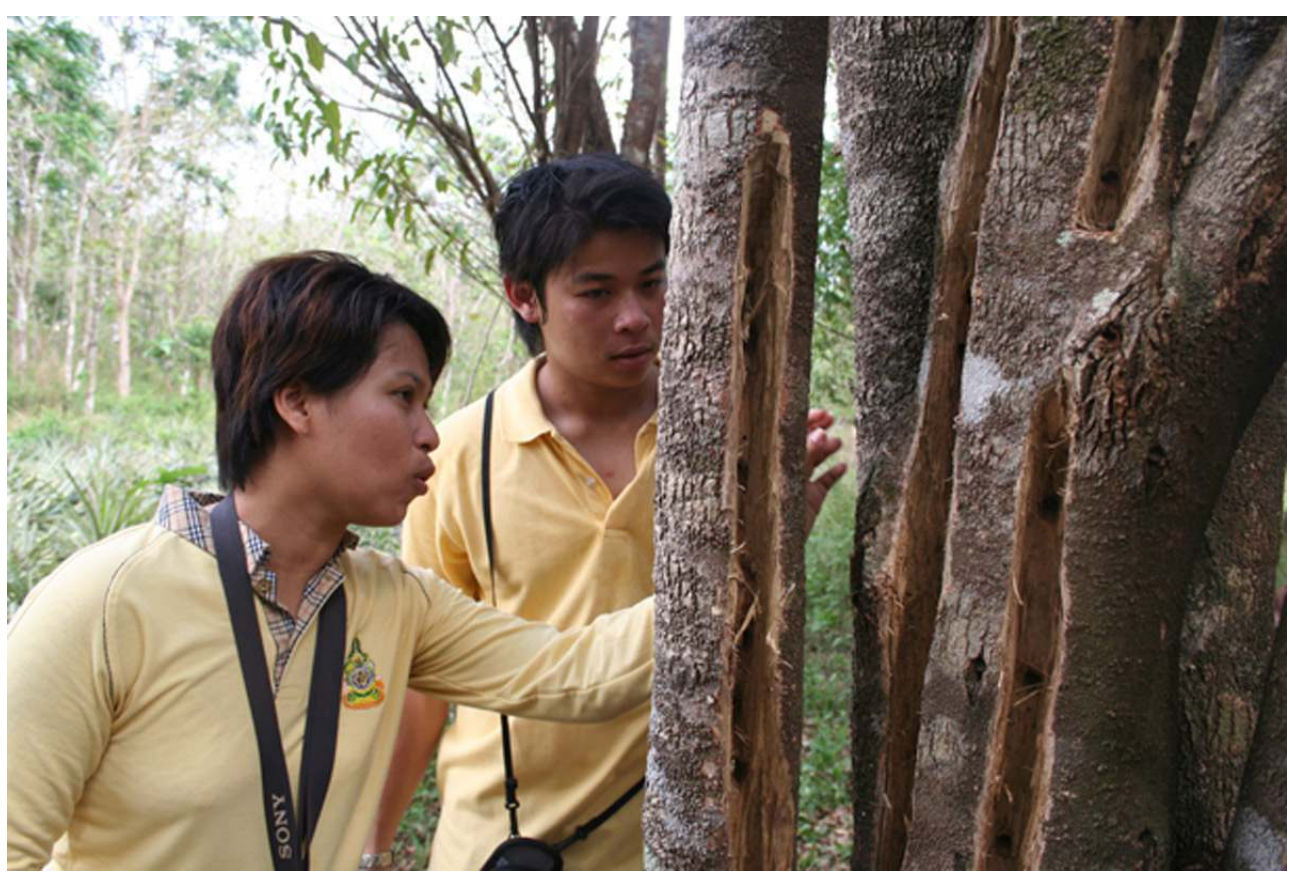

Cliché G.A. Persoon conduit à des tentatives variées de stimulation de son développement. La plus répandue consiste à infliger une blessure délibérée aux arbres à l'aide de grands couteaux ou d'enfoncer des clous dans les troncs.

En général, ces tentatives ne débouchent pas sur des résultats très productifs. Le bois d'aloès est alors d'une qualité inférieure et ne peut être utilisé que pour des usages domestiques. De plus, le bois de haute qualité met des années à se développer. C'est seulement au cours des dernières décennies qu'une démarche plus scientifique a été adoptée dans la culture du bois d'aloès. Des expériences ont été mises en place dans différents pays dont la Chine, la Thaïlande, le Bhutan et l'Indonésie. En Indonésie, par exemple, des plantations ont été établies au Kalimantan oriental, à Lombok, Soumba et en Papouasie. Dans certaines de ces aires, des plantations à relativement grande échelle ont été réalisées comportant des milliers d'arbres d'Aquilaria et bénéficiant d'investissements substantiels dans des unités de transformation. Des entrepreneurs sont attirés par les profits potentiellement élevés que l'on peut faire dans le commerce de cette matière. Cependant, l'un des essais qui ont connu le plus de réussite à ce jour est un projet au Vietnam. Outre des analyses en laboratoire, des expériences sur le terrain ont été développées par une ONG basée à Ho-Chi-Minh-Ville. Le programme « Projet de la Forêt tropicale » (The Rainforest Project, TRP), financé à l'origine par l'Union Européenne, est situé dans l'aire des Sept Montagnes au Vietnam du Sud, non loin de la frontière avec le Cambodge. Les expériences ont été menées avec des agriculteurs locaux et des moines bouddhistes qui avaient acquis une expérience considérable dans la culture de l'Aquilaria, principalement pour leur usage propre. En s'appuyant sur leur savoir, des parcelles expérimentales ont été mises en place pour stimuler la production du bois d'aloès. Le processus et les expériences ont été suivis par un pathologiste du bois. Le principe de base 
du processus a été de percer des trous dans le tronc de l'arbre et de garder la blessure ouverte en introduisant un petit tube en plastique dans le trou. Un traitement chimique a été appliqué sur la blessure pour augmenter le mécanisme de défense et, par conséquent, la production de la résine. Après avoir réalisé des expériences pendant plusieurs années en prenant en compte le nombre de trous, l'âge de l'arbre, la quantité de produits chimiques et d'autres variables, la récolte sur les premiers arbres a eu lieu récemment et la production d'encens fabriqué à partir du bois d'aloès de culture a déjà commencé. La réussite de l'expérience signifie qu'avant peu la méthode s'étendra à d'autres aires où les Aquilaria sont cultivés.

Photo 4. Le " cloutage » est une façon commune dans le Sud-Est de l'Asie pour blesser les arbres d' Aquilaria dans le but de stimuler la production du bois d'aloès

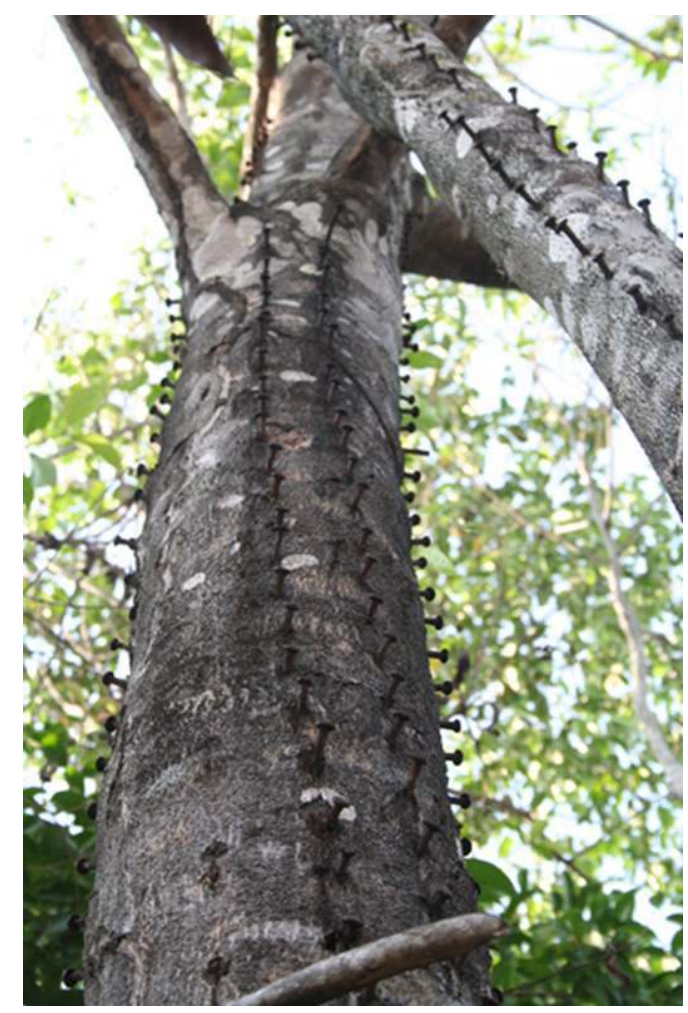

Cliché G.A. Persoon certain nombre de pays. Des plantations ont déjà été établies en Thaillande, en Inde et en Malaisie. De nouveaux arrivants sur la scène sont des hommes d'affaires d'Australie. Ayant acquis une expérience substantielle dans la production du bois de santal en Australie occidentale, des compagnies sont désormais prêtes à s'investir dans les plantations d'Aquilaria, qui pourrait être d'un rapport encore meilleur par unité de production. Cependant, comme cela est souvent le cas pour de nombreuses autres matières précieuses, des produits de pseudo-bois d'aloès déferlent sur le marché. Certains produits, baptisés «Black Magic Wood» (BMW), sont en fait dérivés de bois d'Aquilaria non traité qui a été imprégné d'huile bon marché. Seuls un œil bien exercé et un nez très subtil sont capables de distinguer le véritable bois d'aloès des faux.

Il se pose un certain nombre de questions en relation avec la production de bois d'aloès cultivé à grande échelle. Les prix élevés actuellement atteints par cette matière peuvent- 
ils se maintenir si la production croît substantiellement ? Quelle sera la qualité du produit cultivé ? Des interrogations se sont aussi élevées au sujet des conséquences de la culture à grande échelle pour les producteurs traditionnels de bois d'aloès, les récoltants qui travaillent à l'intérieur des aires forestières. On considère généralement que le bois d'aloès de qualité supérieure naturelle va se raréfier mais que la demande va se maintenir, particulièrement au Japon. Ce segment supérieur du marché ne peut pas être remplacé aussi facilement par le bois cultivé. Quant au bois d'aloès de qualité inférieure, on prévoit une augmentation de la production aussi bien chez les producteurs traditionnels que chez les nouveaux. Une chute graduelle des prix devrait intervenir en raison de l'extension des cultures. Enfin, on estime qu'aura lieu un déplacement progressif de la production, et par conséquent de la valeur, des aires de la forêt tropicale originale vers des plantations établies ailleurs. Des évolutions similaires se sont aussi produites dans le cas d'autres produits forestiers non ligneux, comme les orchidées et le rotin. Dans ce processus, ce sont les lois du marché et du monde des affaires qui dominent. Les producteurs traditionnels de bois d'aloès, comme les moines bouddhistes du Vietnam et de Thaïlande, peuvent être facilement éliminés de la compétition. Leurs récoltes courent le risque d'être victimes d'une rude concurrence par des produits d'une autre origine, parce qu'il n'existe pas encore de marché différencié.

\section{Protection}

18 On discute actuellement de la mise en œuvre de mesures afin d'empêcher la surexploitation des Aquilaria et d'assurer par là leur survie à l'état naturel. Cependant, des porte-parole d'organisations de conservation de la nature désignent une confusion lexicale manifeste comme l'un des principaux obstacles dans ce domaine. Depuis plusieurs années déjà, la Convention sur le commerce international des espèces menacées de la faune et de la flore sauvages (CITES/CCIEM) a listé toutes les espèces d'Aquilaria dans son Annexe II. Ceci implique la nécessité d'en contrôler le commerce (aussi bien importations qu'exportations) (Newton \& Soehartono 2001). Cependant, la confusion lexicale n'est pas aisée à résoudre, parce que le bois d'aloès est connu sous différents noms à travers le monde (comme "eaglewood" ou «bois d'aigle", "agarwood", «jinko», "gaharu» et «oudh»), et parce qu'il est utilisé et même déguisé en de nombreux produits différents (huiles essentielles, parfums, encens, spiritueux, poudre et copeaux de bois). Rechercher les produits à base de bois d'aloès exige des procédures de détection hautement sophistiquées qui n'existent pas encore. C'est là l'une des raisons pour lesquelles on ne peut pas mettre fin facilement au commerce illégal de bois d'aloès. L'un des défis qui se présentent sera d'opérer la différenciation entre le bois sauvage et le bois de culture. Certaines de ces questions doivent être débattues au cours de la prochaine conférence sur le bois d'aloès qui aura lieu dans quelques années. Au cours de la conférence des Parties à la Convention de la CITES qui s'est tenue à La Haye du 2 au 15 juin 2007, des propositions ont été soumises, par l'intermédiaire de la commission des végétaux, dans le but de différencier entre les produits du bois d'aloès sauvage et cultivé. De cette façon, l'exportation et l'importation du bois d'aloès pourraient être mieux régulées. Cependant, ces propositions n'ont pas été adoptées par les Parties du fait du manque de méthodes efficaces de mise en œuvre. 


\section{Conclusion}

19 Il peut sembler surprenant que la chaîne allant de la production à la consommation du bois d'aloès n'ait pas de statut particulier dans le processus menant à sa destination finale. Apparemment, c'est seulement arrivé au stade où il a été transformé en encens qu'il lui est décerné un statut spécial en tant que produit destiné à une pratique rituelle avec une signification symbolique. Il ne lui est pas reconnu de pertinence particulière avant ce stade. Il est considéré comme une marchandise comme une autre et comme une source de profit, alors que le commerce lui-même est hautement spécialisé et nécessite un savoir-faire professionnel considérable en termes de nez, d'appréciation de la qualité et de la transformation. L'histoire de l'exploitation des arbres dont est issu le bois d'aloès ne semble pas différer de celle de produits qui ont également une valeur marchande élevée mais sont de caractère non religieux. Les activités de récolte de ce produit liées à une frontière continuellement mouvante menant à sa surexploitation dominent son histoire.

En tant que tel, il semble être un exemple de type de ressource naturelle dont les perspectives, en termes de protection ou de conditions de production durable, ne sont pas en rapport direct avec sa fonction religieuse ou symbolique. On peut retrouver des relations de nature comparable dans d'autres cas, comme celui de la signification symbolique de la tortue de mer à Bali. Des milliers de tortues de mer étaient tuées dans cette île chaque année, à cause de la nécessité d'offrir des sacrifices rituels, ajoutant dans une large mesure à la surexploitation de la tortue dans les eaux indonésiennes. Une campagne internationale et des discussions sérieuses avec les chefs religieux hindouistes ont permis d'arriver à une réduction importante de l'immolation des tortues, sans avoir à compromettre sa fonction rituelle. Une prise de conscience accrue, parmi les prêtres et les fidèles, des effets sur l'environnement de leurs pratiques religieuses peut contribuer à la protection d'une ressource particulière. Ce souci pourrait aussi être activé en ce qui concerne la situation du bois d'aloès. L'instauration d'une forme de certification pour cette matière produite selon des principes de culture durable pourrait représenter un important pas en avant à cet égard.

21 Et, dans ce sens, la rencontre de tous les acteurs qui jouent un rôle dans l'ensemble de la chaîne de production et de consommation de ce bois peut contribuer à atteindre le but recherché. Appliquer à l'encens fabriqué à partir de bois d'aloès l'effort tendant à « écologiser » la religion semble être un choix évident.

\section{BIBLIOGRAPHIE}

Barden A., Anak N.A., Mulliken T. \& Song M. 2000 - Heart of the matter : Agarwood use and trade and CITES implementation for Aquilaria malaccensis. Cambridge, TRAFFIC International, $60 \mathrm{p}$.

Catholic Encyclopedia 1998 - Incense, http://www.newadvent.org/cathen/07716a.htm. 
Chhetri D.B., Pelden K. \& Dhendup K. 2004 - Sustainable agarwood production through artificial inducement in Bhutan. Council of RNR Research of Bhutan.

Donovan D.G. \& Puri R.K. 2004 - Learning from traditional knowledge of non-timber forest products : Penan Benalui and the autecology of Aquilaria in Indonesian Borneo. Ecology and Society $9(3): 15-32$.

Dunn F.L. 1975 - Rain-forest collectors and trader : A study of resource utilization in modern and ancient Malaya. Monograph 5. Kuala Lumpur, Malaysian Branch Royal Asiatic Society, 150 p.

Groom N. 1981 - Frankincense and myrr : A study of the Arabian incense trade. London, Longman, $285 \mathrm{p}$.

Hansen E. 2000 - The hidden history of a scented wood : aloeswood. Saudi Aramco World 51(6) : 2-13.

Hoskin J. 1994 - Fragrance of the gods. In D. Pratt (Ed.), Incense. Bangkok, Post Book Ltd. : 13-27.

Lawton J. 2004 - Silk, Scents and Spice. UNESCO Publishing / Economica, Paris, 128 p.

Momberg F., Puri R. \& Jessup T. 2000 - Exploitation of gaharu, and forest conservation efforts in Kayan Mentarang National park, East Kalimantan, Indonesia. In Zerner Z. (ed.) People, Plants and Justice: The Politics of Nature Conservation. New York, Columbia University Press : 259-284.

Newton A.C. \& Soehartono T. 2001 - CITES and the conservation of tree species : the case of Aquilaria in Indonesia. International Forestry Review 3(1) : 27-33.

NTFP Exchange Programme 2006 - Eaglewood (Video DVD), Manila.

Pratt D. (Ed.) 1994 - Incense. Fragrance of the gods. Bankok, Post Books Ltd.

Schuitemaker J.P. 1933 - Het garoehout van West Borneo. Tectona, Boschbouwkundig Tijdschrift 26 : 851-892.

Second International Agarwood Conference, Bangkok/Koh Chang, 4-11 March 2007. Organised by The Rainforest Project, FOA, Bangkok, TRAFFIC and Kasetsart University.

Soehartono T. \& Newton A.C. 2001 - Reproductive ecology of Aquilaria spp. in Indonesia. Forest ecology and management $152: 59-71$.

Wollenberg E.K. 2001 - Incentives for collecting gaharu (fungal-infected wood of Aquilaria spp. Thymelaeaceae) in East Kalimantan. Economic Botany 55(3) : 444-456.

Wollenberg E.K. 2003 - Boundary keeping and access to gaharu among the Kenyah forest users. Environment and Planning A 35(6) : 1007-1023.

Zich F. \& Compton J. 2001 - The Final Frontier : Towards Sustainable Management of Papua New Guinea's Agarwood Resource. TRAFFIC Oceania/WWF South Pacific Programme, 11 p.

\section{NOTES}

1. «La Convention sur le commerce international des espèces de faune et de flore sauvages menacées d'extinction, connue par son sigle CITES ou encore comme la Convention de Washington, est un accord international entre États. Elle a pour but de veiller à ce que le commerce international des spécimens d'animaux et de plantes sauvages ne menace pas la survie des espèces auxquelles ils appartiennent » (http://www.cites.org/).

2. Forest Stewarship Council. 


\section{RÉSUMÉS}

Le bois d'aloès, appelé aussi «bois d'aigle ", est un produit forestier non-ligneux très important des forêts d'Asie du Sud-Est. Le bois malade de l'Aquilaria sp. est largement utilisé, aussi bien dans la production d'encens pour les cérémonies religieuses ou le parfum que dans la fabrication de cosmétiques ou de vin médicinal.

En raison de sa grande valeur commerciale, les coupes de bois d'aloès dans les forêts naturelles ont mené l'espèce au bord de l'extinction dans de nombreuses régions du Vietnam, d'Indonésie, du Laos et de Malaisie. Le contrôle de son exploitation et de son commerce est une des raisons qui ont motivé l'inscription de la plante sur la liste II de la CITES. Parallèlement à cette inscription, des mesures d'incitation à la domestication de la plante et à la production de bois infecté ont été lancées. Devant le succès des premières entreprises, d'autres régions se montrent vivement intéressées par la culture des Aquilaria.

Le bois d'aloès est l'un des produits forestiers non ligneux les plus fascinants au monde. C'est un bois infecté par une bactérie de l'arbre Aquilaria, appelé «Le Bois des Dieux » parce qu'il est utilisé comme encens dans des cérémonies religieuses. Le bois d'aloès est un produit très recherché dans de nombreux pays et cultures. Il a une fonction religieuse presque universelle en tant qu'encens, utilisé pour symboliser la purification et accompagner les sacrifices et les prières s'élevant de la terre vers le ciel. L'encens tient une place essentielle dans l'histoire religieuse et le bois d'aloès en est le matériau de base le plus coûteux. On l'utilise depuis les temps anciens. On sait que les Égyptiens et les Grecs s'en servaient dans leurs rites funéraires.

Agarwood, also called eaglewood, is a very important non-timber forest product from the rainforests of Southeast Asia. The infected wood of the Aquilaria sp. is widely used to produce incense for religious ceremonies or for pleasure but it can also be used for making perfumes or medicinal wine.

Due to its high price, the harvesting of agarwood from the wild has brought the plant to the point of extinction in many areas in Vietnam, Indonesia, Laos and Malaysia. That is one of the reasons why this plant has been put on the CITES Appendix II list to monitor its exploitation and trade. At the same time efforts are undertaken to domesticate the plant and stimulate the production of the infected wood. As initial efforts seem to be successful, interested parties from other areas are eager to start growing Aquilaria trees at well.

\section{INDEX}

Mots-clés : bois d'aloès, bois d'aigle, produit forestier non-ligneux, surexploitation, domestication

Keywords : agarwood, eaglewood, non-timber forest product, Southeast Asia, (over) exploitation Index géographique : Asie du Sud-Est, Vietnam, Laos, Indonésie 


\section{AUTEUR}

\section{GERARD A. PERSOON}

Leiden University, Institute Cultural Anthropology and Development Sociology P.O. Box 9555, 2300 RB Leiden.

persoonga@fsw.leidenuniv.nl 